\title{
Return on Investments in Technical Assistance for TB Capacity Building? Factors Influencing Kenyan Health Workers' Performance of TB Skills
}

\author{
Ellen M.H. Mitchell ${ }^{*}, 1,2$, Charlotte E. Colvin ${ }^{3}$, Eveline Klinkenberg ${ }^{1,2}$, Marleen Heus ${ }^{1}$ and Joseph Sitenei ${ }^{4}$ \\ ${ }^{I}$ KNCV Tuberculosis Foundation, The Hague, The Netherlands \\ ${ }^{2}$ Department of Global Health, Academic Medical Center, University of Amsterdam, Amsterdam Institute for Global \\ Health \& Development, The Netherlands \\ ${ }^{3}$ United States Agency for International Development (USAID), Washington D.C., USA \\ ${ }^{4}$ Division of Leprosy Tuberculosis and Lung Disease, Ministry of Public Health and Sanitation, Nairobi, Kenya
}

\begin{abstract}
The dearth of trained personnel to implement TB/HIV services led to substantial investment in human resources and technical assistance in Kenya. Between 1999 and 2006 the staff of the TB program almost doubled. Increases in quantity of TB services occurred, but the impact on quality was unclear.

Analysis of nationally representative data from the 2004 Kenya Service Provisions Assessment (KSPA) of 1,332 TB and/or HIV service providers within 440 public and private health facilities was conducted to compare performance of TB duties between those with and without TB and/or HIV training.

Although the TB-HIV workforce was disproportionately female $(56.9 \%)$, participation in training was less common among women of all cadres (OR $0.4195 \% \mathrm{CI}$.22-.78). After controlling for structural and organizational factors, training in TB diagnosis was strongly associated with performance of smear microscopy (aOR 3.4 95\%CI 1.6-7.3). Mid-level health workers were less likely than doctors to rely on smear microscopy for diagnosis (aOR $0.495 \% \mathrm{CI} 0.2-0.6$ ). Training was associated with direct observation of treatment (D.O.T) (aOR 3.3 CI 1.3-8.9). Other factors positively associated with performance of D.O.T included receipt of supportive supervision (aOR 3.2 CI 2.0-5.0) and an adequate TB drug supply (aOR1.2 95\%CI 1.1-1.4). Barriers included non-alignment with the national directly observed short course policy program (DOTS) (aOR.0.2 95\%CI 0.1-0.7) and working where high volumes of smear microscopy were performed (aOR 0.7 95\%CI 0.5-1.0).

Investments in capacity building including technical assistance during the 2000-2003 period were associated with performance of smear microscopy and directly observed therapy in 2004. However health system factors also influence performance.
\end{abstract}

Keywords: Human resource development, Kenya, Tuberculosis, health worker, capacity building, smear microscopy, training, directed observation of therapy, return on investment, KNCV.

\section{BACKGROUND}

The availability and capacity of human resources to support the expansion of TB/HIV care in Sub-Saharan Africa is often characterized in terms of "a crisis" [1]. The lack of trained personnel to implement priority services has been cited as a key obstacle to achieving the Millennium Development Goals, as well as the Stop TB Partnership's 2015 targets $[1,2]$. Critical gaps remain in the recruitment, capacity building, and retention of staff among every provider cadre as ministries of health work to expand coverage of priority health services, including the classical 5element global TB strategy known as "DOTS" [3]. Long term, sector-wide, comprehensive human resource planning is needed in countries facing shortages, high turn-over and skewed distribution of qualified providers [1]. However,

*Address correspondence to this author at the KNCV Tuberculosis Foundation, P.O Box 176, 2501 CC The Hague, The Netherlands;

Tel: +31 7041672 74; Fax: +31 7035840 04; E-mail: mitchelle@kncvtbc.nl ministries of health, vertical disease management programs, donors, technical assistance partners and other stakeholders rely on short and medium-term capacity building strategies to fill the gap. These include policy reforms, pilot programs and new funding mechanisms that attempt to increase the quantity, quality, distribution and retention of health professionals in Africa [4-6]. Task shifting, emergency hiring, distance learning, telemedicine, rehiring of retired health professionals and public-private mix experiments are underway [6-13].

In 2003, the Global TB Report cited the lack of wellqualified health workers in the 22 high burden countries as the single largest obstacle to meeting the global targets for TB control [14-16]; the shortage is particularly acute throughout Sub-Saharan Africa [17]. The high prevalence of HIV, TB and malaria has had a profound impact on the health work force in the region, increasing burnout, as well as morbidity and mortality $[3,5,18,19]$. In the absence of sector-wide planning, national TB programs (NTPs) have 
implemented short-term in-service training as the key strategy for building human resource capacity for TB control [20], often in settings where TB/HIV collaborative services are being introduced and brought to scale. The best means to integrate TB specific skills in HIV care and other priority health services are widely debated [14, 21-23]. However, pre-service and in-service training remain the classical stopgap approach to ensure that essential services are rendered [20, 24]. Donors and technical assistance partners play a significant role in the design and implementation of training activities.

Despite its ubiquity, the impact of in-service training on TB service delivery is understudied and, the return on investment of this approach is largely unknown [20, 25, 26]. At a time when in-service training for health has come under increasing scrutiny, it is timely to assess its impact on performance of tasks related to TB diagnosis and treatment, as well as TB/HIV integrated services $[16,26]$. Some critics assert that the high volume of single-disease training courses removes front-line health workers from their clinical roles for prolonged periods of time, effectively limiting service provision [5, 22, 27]. Travel reimbursements, "sitting fees" and other inducements to attend training may promote inappropriate trainee selection and limit impact [14, 28]. Due to an overall lack of coordination and the large number of partners engaged in $\mathrm{TB} / \mathrm{HIV}$ training, countries may face challenges in assessing how many staff have been trained to competency in which subjects [14, 28, 29]. Finally, there is concern that intensive training approaches may contribute to "brain drain" to the private sector and foreign countries unless retention measures are taken $[5,12,30]$.

Prior to the advent of The Global Fund and the Presidents' Emergency Plan for AIDS Relief (PEPFAR), Kenya was an early adopter of a comprehensive strategy to address human resource gaps. During the nineteen eighties and early nineties, the Kenyan Government developed a curriculum at the Medical Training College of Nairobi to build TB capacity among clinical officers with the help of KNCV Tuberculosis Foundation (KNCV), The Netherlands Development Organization (SNV), the Foundation of Swiss Civil Servants, and the Netherlands Leprosy Relief Foundation (NLRF) [31]. In 1991 the Governments of Kenya and the Netherlands signed a technical assistance agreement to replace the foreign doctors who had been providing TB care with Kenyan physicians trained in TB. Support from the Dutch government phased out in 2000 and was gradually replaced by other donors. From 2002 to 2006, the United States Agency for International Development (USAID) and the Canadian International Development Agency (CIDA) provided funding for NGOs such as John Snow International (JSI) and KNCV to design and provide technical assistance to implement additional TB training and supportive supervision to health care workers [31]. Combining long, medium, and short term capacity-building approaches on a pilot basis and as well as broader initiatives, The National Leprosy and TB program (NLTP) expanded rapidly from 2000 onwards [31]. Between 2001 and 2003 the TB personnel increased in number from 110 to 140 staff [31, 32]. During this period, the number of diagnostic facilities increased from around 1 to 1.7 facilities per 100,000 population, while the number of health units treating TB increased from 3.4 to 4.4 per 100,000 population [32].
KNCV channeled over US\$350,000 in donor funds to local and international training courses during 2003 alone. There were approximately 1155 health workers and 350 lab personnel trained in TB-related skills in the 2000-2003 period [15]. Performance-related incentives and expedited hiring for staff involved with DOTS implementation, HIV/AIDS prevention, care and treatment programs, and TB/HIV collaborative activities have been instituted in Kenya, although the sustainability of these approaches is still unclear $[10,31,33]$. Similar to other countries in the region, the NLTP has also pursued short-term in-service training as a key component of decentralization, DOTS expansion and the introduction of TB/HIV collaborative activities [34, 35].

The present study focuses on the period prior to 2005, before the advent of the Global Fund and PEPFAR dramatically expanded the breadth and depth of the TB technical assistance landscape to include Kenya Association for Prevention of Tuberculosis (KAPTLD), the International Union Against Tuberculosis and Lung Disease, International Medical Corps, Academic Model for Prevention and Treatment of HIV/AIDS, Program for Appropriate Technology in Health, Family Health International, Malteser Foundation, the African Medical and Research Foundation, Medicine Sans Frontières (MSF), and many other local and international partners [31]. Focusing on the impact of training on service delivery in 2004 makes it possible to explore issues of attribution and the potential impact of partners' technical assistance, if any.

Worldwide, the return on investment for off-site training of clinicians has been highly variable [36-39]. In Kenya, training of community health workers and private providers is common [19, 35, 40-46]. Improvements in diagnostic or prescribing practices have been reported following training, but patient treatment outcomes are infrequently linked to investments in capacity building $[19,37]$.

Given the prominence and resources invested in short term, in-service training strategies promoted by international technical assistance partners, program managers, policy makers, and donors need to know whether TB training exposures result in improved provider performance and ideally patient outcomes [26]. However, the specific impact of training on provider clinical behavior is difficult to measure due to the array of factors that influence provider performance [47]. Evaluators rarely have the opportunity to utilize proper baseline measures, comparison groups, or analyses that adjust for all the potential influences on performance. Thus, most evaluations of different human resource development approaches measure only short-term knowledge acquisition, attitudinal change, or behavioral intentions but do not measure skill utilization [47].

Randomized trials and quasi-experimental studies examining the effect of in-service training on the clinical practices of health professionals have generally shown significant impacts from interactive courses, involving hands-on skills practice, reinforced by supportive supervision [48-50]. However, a cluster randomized control trial undertaken in South Africa in the context of training on DOTS implementation showed no effect on TB patient outcomes [51]. The effects of training in DOTS implementation have been explored in the context of publicprivate mix approaches in urban Africa [52], where the 
impact of training on private health workers' behavior is more difficult to measure and thus even less well understood than public sector training programs [53].

To assess the potential association between participation in TB training activities between 2000- 2003 and essential health worker behaviors, (e.g. performance of smear microscopy, direct observation of treatment, and provision of IPT for HIV+ clients) we performed secondary analysis of the 2004 Kenya Service Provision Assessment data.

\section{CONCEPTUAL MODEL}

International technical assistance was a catalyst in the design, organization, and implementation of large scale TB training efforts which intensified from 2000 onwards with the influx of technical and financial support from USAID, $\mathrm{CDC}, \mathrm{KNCV}$, and Canadian CIDA.

Key factors hypothesized to influence the relationship between investments in training and health worker performance can be defined in three domains: 1) Provider characteristics (e.g. cadre, gender, age, prior experience providing TB diagnosis and treatment services, education); 2) Structural factors (e.g. facility complexity, availability of TB diagnostic and treatment commodities, demand for TB services); and 3) Organizational context (e.g. public or privately managed, policies support for direcly observed therapy (DOT), range of duties, promotion opportunities, incentives, quality of supervision) [7].

Fig. (1) illustrates the hypothesized relationships between investments in TB and HIV training, the three domains influencing provider performance, and provision of best practices related to implementation of DOTS at the facility level. Self-reported provision of TB prevention, diagnosis and treatment services by the health care worker is modeled as a function of individual acquisition of skills via in-service training. We posited that any effects of training on provision of services would be mediated by provider characteristics, available infrastructure, and organizational context.

Provider characteristics influencing provision of services were hypothesized to be: number of years of education and experience as a provider, age, gender and cadre [7]. These attributes are often collinear.

Infrastructural factors thought to modify the effect of training on provider performance include operating authority, facility type, TB diagnostic and treatment capacity, and range of services provided at the facility [54]. We hypothesized that some facility types may be more likely than others to provide a conducive environment for TB skills utilization following training. For example, hospitals with on-site smear microscopy may favor use of microscopy. Similarly, the presence of an adequate drug supply could also be assumed to favor the provision of directly-observed therapy and isoniazid preventive therapy (IPT).

Organizational factors thought to influence performance of recommended TB tasks include facility participation in the national DOTS program, degree of cross-referral within TB/HIV/STI (sexually transmitted infection) services, the availability of promotional opportunities, performance incentives, and quality supervision of health workers $[7,10$, 55]. In many studies, performance audits with feedback have been shown to boost health worker performance $[7,56,57]$.

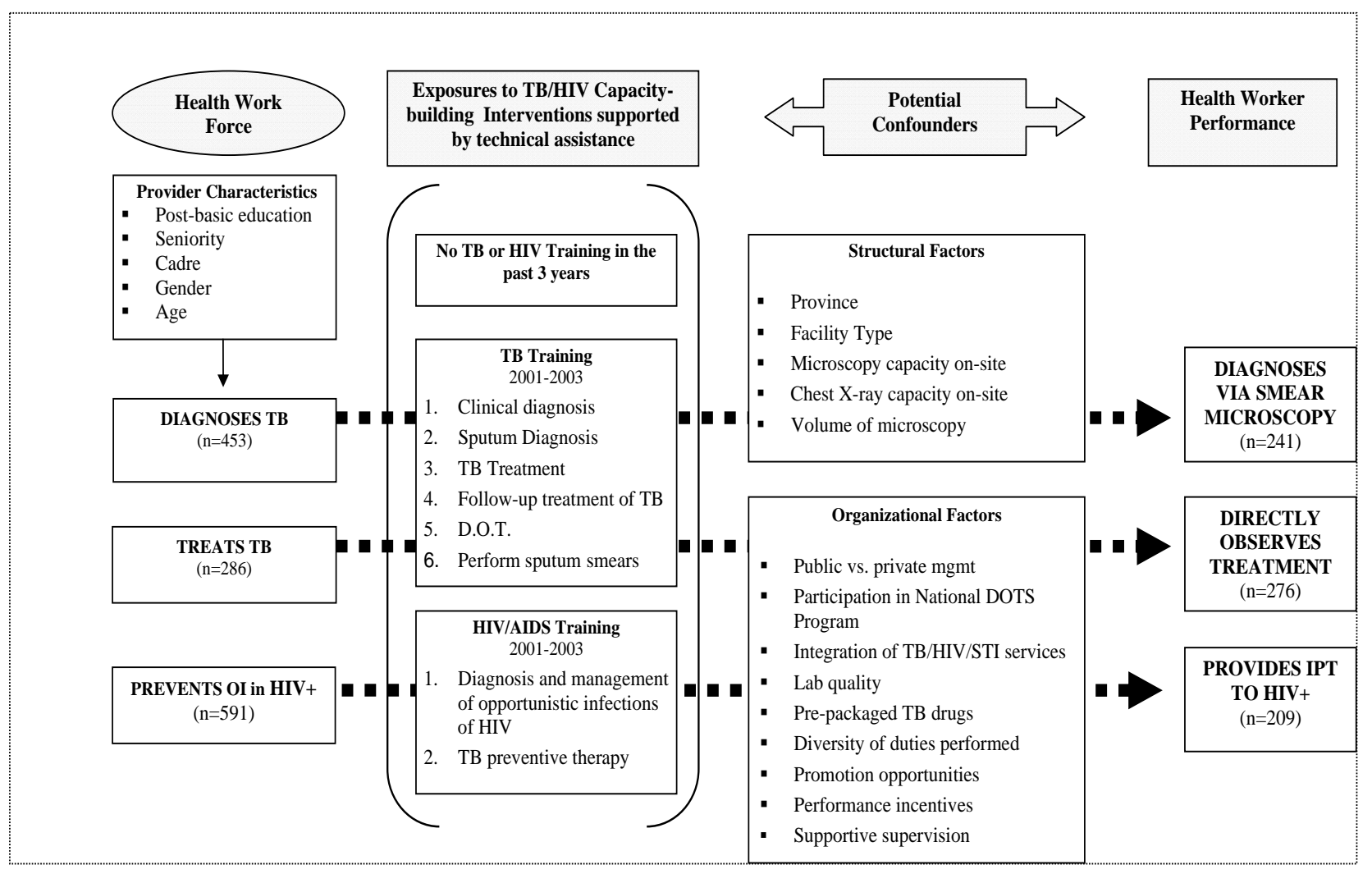

Fig. (1). Conceptual Framework. 


\section{METHODS}

The 2004 Kenya Service Provisions Assessment (KSPA) is a nationally representative survey of 2,081 health workers within 440 public and private health care facilities in Kenya. The 2004 KSPA was designed with input from the Division of Leprosy, Tuberculosis and Lung Diseases and was implemented by the National Coordinating Agency for Population and Development (NCAPD) with support from the Ministry of Health, the University of Nairobi, and the Central Bureau of Statistics and ORC Macro through the USAID MEASURE DHS project (Demographic Health survey).

Sites were drawn with replacement from a master list of all public, private-for-profit, NGO and FBO health facilities. Data collection included two components: a facility inventory questionnaire identifying commodities, policies, management systems, and infrastructure to support service provision, and a health provider interview exploring the qualifications, training, performance and perceptions of health workers. A health service provider was defined as one who provides consultation services, counseling, health education, or laboratory services to clients. If an individual only took measurements or completed registers and never provided any type of professional health services, they were excluded.

Facilities were selected with proportional representation to the number of facilities in each province; however, sites offering HIV services were oversampled to ensure sufficient data for analyses. A quota sample of 8 health workers per site was randomly drawn among all providers present at a selected facility on the day of the survey. In facilities with fewer than eight health service providers, all consenting staff was interviewed. Sampling weights were used to adjust for probable over-sampling or under-sampling of particular cadres within facility type and province and to account for intentional oversampling of HIV services. The survey methodology has been previously described [58].

Health workers were only included in the current analysis if they reported providing either TB clinical services or preventive care for HIV+ clients. The sample therefore included all health workers who diagnose TB or opportunistic infections (OI) of HIV, treat TB or OI of HIV, and prevent $\mathrm{OI}$ in $\mathrm{HIV}+$ clients. Health workers were surveyed on their participation in training courses over a three year interval (2002-2004) prior to the survey. Providers who diagnosed TB were recoded into those who rely on smear microscopy and those who never utilized smear microscopy as a basis for TB diagnosis. Health workers who treated TB were divided into those who reported directly observing patients' therapy and those who did not ever directly observe therapy. Finally those who reported that they prevented opportunistic infections in HIV+ clients as part of their jobs were divided into those who provided INH preventive therapy for HIV+ clients and those who never provided IPT. Provider characteristics such as gender, age, years of post-secondary education, profession, and length of TB service were collapsed into dichotomous categories or means for the purposes of analysis.

\section{Training Exposures}

A dichotomous diagnostic training variable was created combining the affirmative answers to any of 5 specific queries on course content on diagnosis services. A dichotomous TB treatment training variable was similarly derived from any positive responses to 4 queries on treatment content. Two training exposures, the lung diploma course and the 3-week IUATLD course (International Union Against Tuberculosis and Lung Disease), were treated as both diagnostic and treatment training exposures. A TB prevention training variable was created from those who reported being trained in "preventive treatment for TB". A continuous variable was derived representing the cumulative exposure to any of 10 possible TB training exposures (a national diploma for chest and skin, an IUATLD course, a TB treatment course, a sputum diagnosis course, an IPT adherence counseling course, diagnosis of opportunistic infections course, management of opportunistic infections course, preventive treatment for TB (IPT) course, sputum test course, or any other TB diagnostic course). In light of the absence of detailed information on each training, the cumulative training variable was an exploratory one.

\section{Organizational Factors}

A supervision score was generated from the unweighted responses to 8 questions about whether a health worker had been personally supervised and whether supervision included: delivery of supplies, checking records or reports, observation of work, feedback (positive or negative), encouragements, written comments, updates on technical or administrative issues or discussion of work-related problems. An unweighted incentive score was created to reflect the sum of all incentives, including: receipt of salary supplements, non-monetary incentives, and/or opportunities for promotion.

\section{Structural Factors}

To measure laboratory capacity, an unweighted summary score of 12 laboratory quality measures was created. The score was composed of a sum of these items: presence of a TB lab manual, a functional microscope, functional refrigerator, functional incubator(s), test tubes, glass slides and covers, AFB (acid-fast bacilli) or Ziehl-Nielson stains, TB culture services, other TB tests, TB lab notebook with results, and evidence of accurate record keeping. A TB drug commodity score was similarly calculated as the crude sum of eight in-stock TB medicines, including isoniazid (INH). The annual volume of smears analyzed in each facility was recoded and each facility's smear microscopy processing load was categorized as either low (0-100), medium (101$500)$, high $(501-1,500)$, or very high $(1,501-10,000)$.

Where required by the questionnaires, the research assistant indicated whether a specific item being assessed was "observed", reported to be "available but not observed", "not available," or it was "uncertain" whether the item was available. Equipment, supplies, registers, and manuals for TB services were defined as 'available' only if they were in the relevant TB service delivery area or in an immediately adjacent room. 
Variables were recoded in SPSS16.0 and transferred into STATA 10.0 to adjust for multistage sampling design using the SVY command. Bivariate analyses tested for associations between performance of smear microscopy, DOT, and IPT and proxies for the three domains that may mediate training performance (health worker characteristics, infrastructure, and organization) hypothesized in the conceptual framework. The normality and equal variance assumptions were checked for the two-sample t-tests for continuous variables. Continuous variables are presented as means with standard errors (SE). Variables significant at 0.1 level in univariate analysis were entered in the multivariate models. All results referred to in the text are significant at the 0.05 level unless stated otherwise.

\section{Ethical Considerations}

Data collectors were trained to observe ethical guidelines (e.g., maintain confidentiality, anonymity if pertinent, and not to intervene during service delivery). Participation was voluntary and respondents could refuse to answer individual questions or skip entire sections without penalty. Written informed consent was obtained from the facility in-charge and from all respondents for the facility inventory questionnaires. As this study is based on secondary analysis of an anonymous dataset generated by the Central Bureau of Statistics in 2004, repeat ethical review was not deemed essential for the protection of human subjects. Permission to conduct this analysis was sought and obtained from the organizations that collected the data.

\section{RESULTS}

\section{Sample Characteristics}

\section{Demographic Characteristics, Occupational and Workload} Characteristics

A total of 1,332 health workers (providing either TB clinical services or preventive care for HIV+ clients) in 283 facilities met the criteria for inclusion. TB/HIV care providers include a wide range of professionals, but predominantly mid-level staff (nurses and midwives) (81\%). More than half (56.9\%) were female and nearly half $(49.5 \%)$ were between 31 and 45 years old, with $29.4 \%$ and $29.9 \%$ in the younger (21-30 years) and older (46-71 years) categories, respectively (Table 1). The average provider was in his/her current position for almost seven years and had about six months of post-basic education. Over half of providers worked in hospitals $(52.3 \%)$ and a majority worked in facilities managed by the government $(61.9 \%)$. In terms of clinical duties, TB or HIV care providers offered an average of 14 clinical services as part of their routine work.

\section{Performance of TB Standard Diagnostic and Treatment Practices}

More than half of the study sample $(55.1 \%)$ provided HIV care to prevent opportunistic infections and a slightly smaller proportion (48.4\%) provided clinical TB services (Table 1), suggesting that in 2004 there was considerable verticality with very few staff providing both TB and HIV care. Just over one third (35.5\%) reported reliance on clinical algorithms for TB diagnosis and a smaller proportion $(22.5 \%)$ reported reliance on smear microscopy to diagnosis TB. One third (33.8\%) provided follow-up care for TB patients and $26.7 \%$ provided TB treatment, while almost the same proportion $(25.7 \%)$ specifically offered directly observed treatment.

\section{Exposure to TB-Related Training}

Roughly a quarter of the respondents $(21.6 \%)$ reported receiving some type of $\mathrm{TB}$ training in the previous three years; more reported receiving TB diagnosis and treatment training $(23-25 \%)$ than TB prevention training $(9.5-11 \%)$. Only $2.2 \%$ of $\mathrm{TB}$ service providers reported receiving per diems in exchange for attending trainings. Male doctors (55.3\% vs 39.7\%; OR 1.9, CI: 1.1-3.2) and male mid-level providers (47.3\% vs 35.8\%; OR, $1.6 \mathrm{CI}$ : $1.1-2.4)$ were more likely than female staff to have attended TB training.

\section{Service Delivery Environment}

Almost two thirds of TB or HIV care providers (62.1\%) worked in a site that participated in the national DOTS program and just over one third (34.6\%) worked in a facility with X-ray services available (Table 2). Almost one quarter $(24.1 \%)$ reported availability of pre-packaged TB treatment at their site. Smear microscopy was generally available at the workplace, with almost two thirds of the facilities $(62.7 \%)$ conducting tests on-site. However, $18.8 \%$ reported referral of suspects to another facility for this service and $3.4 \%$ collected specimens and sent them for testing. The volume of smear microscopy processed varied among the facilities with near equal proportions of facilities processing $<100$ slides (27.8\%), 101-500 (22.9\%), 501-1,500 (23\%), and over 1,500 slides $(26.3 \%)$ in the previous year. Almost two thirds $(63.9 \%)$ of respondents received a supervisory visit in the 6 months prior to the survey.

\section{Factors Influencing the Use of Smear Microscopy for Diagnosis}

Having received training in $\mathrm{TB}$ diagnosis was weakly associated with providers' use of smear microscopy at the bivariate level $(65.5 \%$ vs $48.4 \% \mathrm{p}=08)$ (Table 3$)$. Training in the diagnosis of opportunistic infections for HIV had no impact on the use of microscopy, however the number of health workers exposed to IPT training for HIV+ clients prior to 2004 was small $(n=100)$. As expected, structural factors played an important role enabling use of smear for diagnosis. Understandably the presence of smear microscopy on-site $(59.2 \%$ vs $31.1 \%)$ and the mean volume of slides reviewed annually (1280 vs 774) were both positively associated with use of smear. However, $40.7 \%$ of health workers with on-site access to smear microscopy did not avail it when making a TB diagnosis. Surprisingly, organizational factors were not critical determinants of whether health staff used microscopy for diagnosis, with the curious exception of performance incentives. The more performance incentives that a health worker received, the less likely he/she was to use microscopy for diagnosis (although the mean number of incentives received were small in both groups). Although gender, age, and years of experience in TB care were not associated with use of microscopy, being a physician as opposed to a midlevel health worker (65.4\% vs 48.8\%) increased reported use of smear microscopy.

In the final model, exposure to $\mathrm{TB}$ training was positively associated with use of smear microscopy 
Table 1. Sample Characteristics

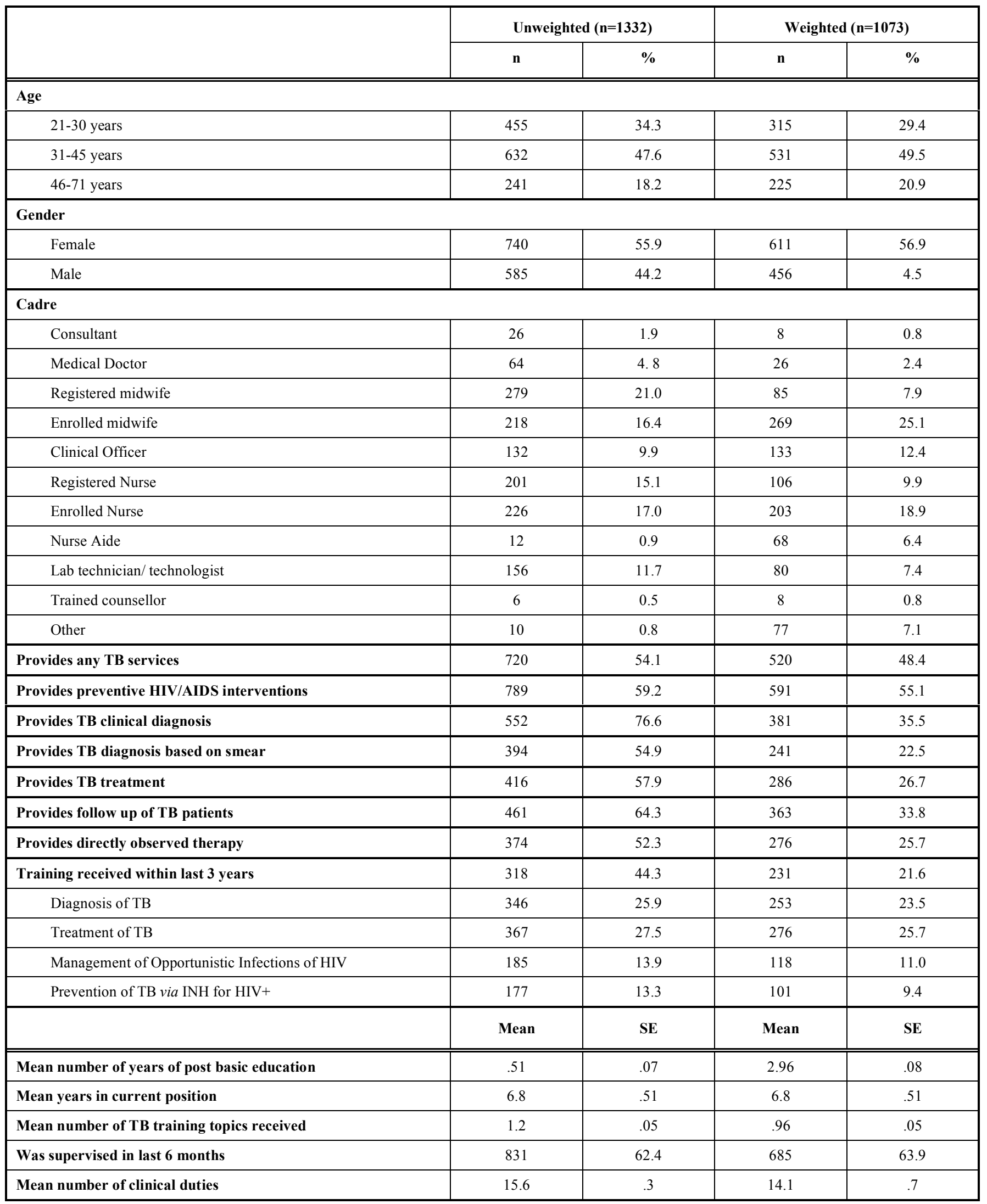


Table 2. Service Delivery Environment for TB and HIV Staff

\begin{tabular}{|c|c|c|c|c|}
\hline \multirow[b]{2}{*}{ Operating authority } & \multicolumn{2}{|c|}{ Unweighted $(n=1332)$} & \multicolumn{2}{|c|}{ Weighted $(n=1073)$} \\
\hline & n/Average & $\%(\mathrm{SE})$ & n/Average & $\%(\mathrm{SE})$ \\
\hline Government & 691 & 51.9 & 664 & 61.9 \\
\hline Non-government & 641 & 48.1 & 409 & 38.1 \\
\hline \multicolumn{5}{|l|}{ Facility Type } \\
\hline Hospital & 913 & 68.5 & 562 & 52.3 \\
\hline Clinic & 95 & 7.1 & 7 & 0.7 \\
\hline Health centre & 141 & 10.6 & 282 & 26.3 \\
\hline Dispensary & 66 & 4.9 & 159 & 14.8 \\
\hline Maternity & 102 & 7.7 & 58 & 5.4 \\
\hline Stand alone VCT & 15 & 1.1 & 7 & 0.6 \\
\hline Has $x$ ray on site & 521 & 39.1 & 372 & 34.6 \\
\hline Site participates in national DOTS program & 882 & 66.2 & 666 & 62.1 \\
\hline Site pre-packaged TB drugs & 317 & 23.8 & 258 & 24.1 \\
\hline \multicolumn{5}{|l|}{ Site smear microscopy policy } \\
\hline Microscopy conducted on-site & 934 & 71.2 & 673 & 62.7 \\
\hline Specimens collected, sent elsewhere & 61 & 4.7 & 36 & 3.4 \\
\hline Client sent elsewhere for microscopy & 220 & 16.8 & 202 & 18.8 \\
\hline Microscopy not used for diagnosis & 96 & 7.3 & 132 & 12.3 \\
\hline Lab commodity score & 8.6 & $(.03)$ & 8.4 & $(.13)$ \\
\hline \multicolumn{5}{|l|}{ Annual demand for sputum tests in facility } \\
\hline $0-100$ tests & 251 & 25.9 & 207 & 27.8 \\
\hline $101-500$ tests & 288 & 29.8 & 170 & 22.9 \\
\hline $501-1500$ tests & 238 & 24.6 & 171 & 23.0 \\
\hline $1501+$ tests & 191 & 19.7 & 195 & 26.3 \\
\hline
\end{tabular}

(aOR 3.4, CI 95\%: 1.6-7.3) (Table 4). Being a nurse or midwife was associated with reduced use of smear microscopy (aOR 0.4, CI: 95\% 0.2-0.6).

\section{Factors Associated with Provision of Directly Observed Therapy}

Training on TB treatment or treatment of opportunistic infections of HIV was associated with provision of directly observed therapy (DOT) (Table 5). On average, health workers who reported providing DOT were slightly more likely to have attended more TB trainings, than those who did not employ DOT when treating patients (mean of $2.0 \mathrm{vs}$ 1.3 trainings). Structural factors played a limited role in health workers' implementation of DOT. Health workers employed within facilities processing a high volume of smear microscopy slides (i.e. hospitals) were less likely to directly observe TB patients' treatment. Organizational factors were critically important for the performance of DOT. Health workers within facilities participating in the national DOTS program were more likely to implement DOT $(64.5 \% \mathrm{v} 38.5 \%)$. Health workers in facilities that offered TB testing for clients availing the STI clinic were also more likely to employ DOT for patients. Health workers were more likely to perform DOT for patients if they worked in facilities with a higher mean TB drug supply score (4.4 vs 3.2). Finally, although provision of supportive supervision was limited among all TB/HIV workers, those who reported marginally higher quality supervision ( 0.9 vs 0.7 scores). were more likely to perform DOT. Finally, mid-level providers were more likely to directly observe TB patients than physicians or clinical officers $(63 \%$ vs $41 \%)$.

In the final multivariate model, receipt of training in TB treatment was the most significant predictor of use of DOT (Table 6). Training also had a cumulative effect on DOT provision, for each additional type of training received, health workers' were $20 \%$ more likely to use DOT. Receiving supportive supervision was also a significant determinant of performance of DOT (OR 3.2, 95\% CI: 2.05.0). Working in a facility with a stable TB drug supply also favored use of DOT (OR 1.2, 95\% CI: 1.1-1.4). The barriers to carrying out DOT were identified as working in a facility that did not participate in the national DOTS program and working in a facility where a high-volume of smear microscopy is performed (e.g. tertiary referral hospitals). 
Table 3. Factors Influencing the Diagnosis of TB Using Smear Microscopy ( $\left.{ }^{*} n=517\right)$

\begin{tabular}{|c|c|c|c|c|c|}
\hline & \multicolumn{5}{|c|}{$\frac{\text { Diagnoses TB }}{n^{*}=454}$} \\
\hline & \multicolumn{2}{|c|}{ Uses Smear Microscopy $\left(n^{*}=241\right)$} & \multicolumn{2}{|c|}{ Does Not Use Smear Microscopy $\left(n^{*}=213\right)$} & \multirow{2}{*}{$p$ Value } \\
\hline & $\mathrm{n}^{*}$ & $\%(\mathrm{SE})$ & $\mathrm{n}^{*}$ & $\%(\mathrm{SE})$ & \\
\hline \multicolumn{6}{|c|}{ Training Exposures } \\
\hline \multicolumn{6}{|l|}{ Training in TB diagnosis } \\
\hline No & 159 & 48.4 & 170 & 51.6 & 0.08 \\
\hline Yes & 82 & 65.5 & 43 & 34.5 & \\
\hline Mean TB Training Exposure Score & 1.9 & $(.17)$ & 1.4 & $(.20)$ & 0.22 \\
\hline \multicolumn{6}{|c|}{ Structural Factors } \\
\hline Facility Type & & & & & 0.16 \\
\hline Hospital & 145 & 58.9 & 101 & 41.1 & \\
\hline Non-hospital & 96 & 46.4 & 111 & 53.6 & \\
\hline Chest Radiograph Technology on-site & & & & & 0.39 \\
\hline No & 130 & 49.8 & 131 & 50.2 & \\
\hline Yes & 111 & 57.8 & 81 & 42.2 & \\
\hline Sputum Microscopy performed on-site & & & & & 0.003 \\
\hline No & 31 & 31.1 & 68 & 68.6 & \\
\hline Yes & 210 & 59.2 & 145 & 40.7 & \\
\hline Mean Number of sputum tests performed annually & 1280 & $(175.2)$ & 774 & $(137.4)$ & $<0.001$ \\
\hline \multicolumn{6}{|c|}{ Organizational factors } \\
\hline Operating Authority & & & & & 0.17 \\
\hline Governmental & 152 & 43.4 & 198 & 56.6 & \\
\hline Non-governmental & 89 & 53.2 & 78 & 46.8 & \\
\hline Lab Quality Score & 7.3 & $(.25)$ & 6.0 & $(.42)$ & 0.06 \\
\hline Medicine Commodity Score & 4.0 & $(.24)$ & 3.7 & $(.24)$ & 0.83 \\
\hline Supervision score & 0.8 & $(.03)$ & 0.9 & $(.04)$ & 0.20 \\
\hline Number of clinical duties & 16.3 & $(1.2)$ & 14.8 & $(1.2)$ & 0.50 \\
\hline Performance Incentives & 1.1 & $(.07)$ & 1.4 & $(.09)$ & 0.03 \\
\hline \multicolumn{6}{|c|}{ Provider characteristics } \\
\hline Gender & & & & & 0.36 \\
\hline Male & 133 & 56.8 & 101 & 43.2 & \\
\hline Female & 106 & 48.8 & 161 & 51.2 & \\
\hline Cadre & & & & & .01 \\
\hline Physician/Clinical Officer & 88 & 65.4 & 46 & 34.6 & \\
\hline Other providers & 144 & 46.5 & 166 & 53.5 & \\
\hline Years delivering TB care & & & & & 0.94 \\
\hline$<5$ years & 114 & 47.7 & 108.8 & 51.3 & \\
\hline$\geq 5$ years & 125 & 52.2 & 103 & 48.6 & \\
\hline Age in years & 35.0 & $(.9)$ & 34.9 & (1.4) & 0.50 \\
\hline Years of post-basic education & 2.9 & $(.09)$ & 2.9 & $(.12)$ & 0.53 \\
\hline Years providing $\mathrm{TB}$ care & 7.5 & (.68) & 6.4 & $(.94)$ & 0.21 \\
\hline
\end{tabular}


Table 4. Multivariate Analysis of Predictors of Use of Smear Microscopy

\begin{tabular}{|l|c|c|c|}
\hline & Adjusted Odds Ratio & $\mathbf{9 5 \%}$ C. I. & $\boldsymbol{p}$ Value \\
\hline \hline Training in TB diagnosis & $\mathbf{3 . 4}$ & $\mathbf{1 . 6 - 7 . 3}$ & $\mathbf{0 . 0 0 2}$ \\
\hline Sputum microscopy performed on site & 0.3 & $0.1-1.3$ & 0.112 \\
\hline Mean number of sputum tests performed annually & 1.1 & $0.9-1.0$ & 0.192 \\
\hline Incentive Score & 0.7 & $0.5-1.1$ & 0.125 \\
\hline Cadre: Mid-level & $\mathbf{0 . 4}$ & $\mathbf{0 . 2 - 0 . 6}$ & $\mathbf{0 . 0 0 1}$ \\
\hline
\end{tabular}

Text in bold represent statistically significant findings.

\section{Factors Influencing Provision of Isoniazid Preventative Therapy (IPT)}

In univariate analysis staff trained in prevention of TB in HIV positive patients was more likely to provide IPT (53\% vs 32\%) (Table 7). Structural factors also favored the provision of IPT, (e.g. being a hospital and having chest radiography performed on site). Participation in the national DOTS program, provision of microscopy to STI clients, availability of pre-packaged TB drugs, higher lab quality, and medicine commodity scores all favored provision of IPT. Physicians and clinical officers were more likely than other providers to provide IPT $(48 \%$ vs $32 \%, \mathrm{p}=0.02)$. Each additional year of post-basic education favored the provision of IPT. Staff with more years of experience either in diagnosing or treating opportunistic infection in HIV positive persons more often provided IPT. If experience providing TB diagnosis and treatment was 5 years or more, over $40 \%$ provided IPT while this was just about a quarter in those with less than 5 years of TB experience.

In the multivariate model, none of these factors remained positively associated with provision of IPT (Table 8). Possibly this is a function of collinearity among them, as many were characteristics of doctors operating in hospitals. Alternatively, it may have been a function of the small number of observations, as few health workers (9.4\%) reported having been trained in IPT for HIV+ clients in 2004.

\section{DISCUSSION}

Investments in technical assistance to support training of Kenya's health workers in smear microscopy for diagnosis and DOT for treatment appear to yield significant return on investment. Although female health workers represent the majority of the TB/HIV work force in 2004, male health workers of all cadres attended more TB training. It is not possible to know if this due to female workers opting out or if TB capacity-building opportunities were inequitably distributed. Nurses and midwives represent almost $70 \%$ of the self-identified TB health workforce, but they were less likely to rely upon smear microscopy.

Although reported receipt of supportive supervision was low, it was found to be strongly associated with increased provision of directly observed therapy (OR 3.2 CI 95\% 2.05.0), and should reinforce efforts to improve individual health worker performance through regular visits and feedback.

Given the dual practice of many clinicians and Kenya's significant investments in private sector TB provision, it is not surprising that health workers in public sector facilities were not significantly better in their performance of these three key skills than private sector clinicians [59].

It is possible that the differential impact of training on performance measured here may not be a reflection of the quality of the education, but rather the current division of labor. Anecdotally it has been suggested that in some facilities trained mid-level providers are not authorized to diagnose all forms of TB (e.g. smear negative) or to rule out TB in HIV+ clients. Their reduced reliance on smear microscopy may also be a function of the greater proportion of pediatric patients they treat. The fact that working in a setting with a busy smear microscopy lab is associated with less implementation of directly observed therapy suggests that tertiary care settings may not be optimally integrated into national TB programs. Finally, there was no policy on IPT for HIV+ clients until 2009 in Kenya and very limited training on this important topic. Therefore it is understandable that provision of IPT for HIV+ clients was very low in $2004(35.3 \%)$ and was not positively influenced by training $(9.4 \%)$ in prevention of opportunistic infections and/or Isoniazid prophylaxis. Moreover, after controlling for confounding in the final model, it was no longer influenced by structural or organizational factors.

\section{LIMITATIONS}

An important limitation of this analysis is the lack of robust measures of the depth and quality of TB training interventions beyond the proxy variable "length of training" which was available for only a very small proportion of training courses. Similarly, the method of trainee selection is a potential confounder that was not measured in the survey. A second limitation of this study is its cross-sectional design which does not account for any delayed benefit of training on performance or decay in performance over time. A third limitation is that the principal exposures and outcomes (performance of best practices) are self-reported and may be subject to social desirability bias. Finally, health workers who are trained may share useful knowledge and skills with untrained peers. As such, some leakage (and hence misclassification) may have occurred that may lead to an underestimate of the magnitude of the positive effect of training and technical assistance.

\section{CONCLUSIONS AND RECOMMENDATIONS}

TB training interventions in the 2000-2004 periods appeared to be significantly associated with the performance of smear microscopy and direct observation by Kenyan health 
Table 5. Factors Associated with Performance of DOT ( $\left.{ }^{*}=456\right)$

\begin{tabular}{|c|c|c|c|c|c|}
\hline & \multicolumn{5}{|c|}{$\frac{\text { Follows Up TB patients }}{n^{*}=456}$} \\
\hline & \multicolumn{2}{|c|}{$\begin{array}{l}\text { Directly Observes Treatment } \\
\qquad\left(\mathrm{n}^{*}=276\right)\end{array}$} & \multicolumn{2}{|c|}{$\begin{array}{l}\text { Does Not Directly Observe Treatment } \\
\qquad\left(\mathrm{n}^{*}=180\right)\end{array}$} & \multirow[t]{2}{*}{$p$ Value } \\
\hline & $\mathbf{n}^{*}$ & $\%$ (se) & $\mathbf{n}^{*}$ & $\%(\mathrm{se})$ & \\
\hline \multicolumn{6}{|c|}{ Training Exposures } \\
\hline Trained in TB Treatment and/or management of OI in $\mathrm{HIV+}$ & & & & & 0.027 \\
\hline No & 111 & 49.5 & 114 & 50.5 & \\
\hline Yes & 133 & 66.8 & 66 & 33.2 & \\
\hline Mean TB Training Exposure Score & 2.0 & $(.19)$ & 1.3 & $(.15)$ & 0.007 \\
\hline \multicolumn{6}{|c|}{ Structural Factors } \\
\hline Facility Type & & & & & 0.51 \\
\hline Hospital & 147 & 60.0 & 98 & 40.0 & \\
\hline Non-hospital & 97 & 54.1 & 82 & 45.9 & \\
\hline \multicolumn{6}{|l|}{ Sputum Microscopy performed on-site } \\
\hline No & 44 & 46.3 & 51 & 53.8 & 0.11 \\
\hline Yes & 200 & 60.9 & 129 & 39.1 & \\
\hline Mean number of sputum tests performed annually & 1027 & $(169.2)$ & 1490 & $(243.2)$ & $<0.001$ \\
\hline \multicolumn{6}{|c|}{ Organizational Factors } \\
\hline Operating Authority & & & & & 0.42 \\
\hline Governmental & 175 & 59.7 & 118 & 40.3 & \\
\hline Non-governmental & 69 & 52.7 & 62 & 47.3 & \\
\hline Facility participates in National DOTs program & & & & & 0.008 \\
\hline No & 44 & 38.5 & 70 & 61.4 & \\
\hline Yes & 200 & 64.5 & 110 & 35.5 & \\
\hline TB testing provided to STI clients & & & & & 0.046 \\
\hline No & 45 & 43.2 & 59 & 56.8 & \\
\hline Yes & 199 & 62.3 & 120 & 37.7 & \\
\hline Medicine Commodity Score & 4.4 & $(.19)$ & 3.2 & $(.24)$ & $<0.001$ \\
\hline Pre-packaged Drugs for Patients & & & & & 0.63 \\
\hline No & 179 & 56.6 & 137 & 43.7 & \\
\hline Yes & 65 & 60.7 & 42 & 39.3 & \\
\hline Supervision score & 1.0 & $(0.03)$ & 0.7 & $(0.05)$ & $<0.001$ \\
\hline Number of clinical duties & 15.5 & $(1.52)$ & 16.5 & $(0.72)$ & 0.5 \\
\hline Performance Incentives & 1.3 & $(0.10)$ & 1.2 & $(0.08)$ & 0.5 \\
\hline \multicolumn{6}{|c|}{ Provider Characteristics } \\
\hline Gender & & & & & 0.37 \\
\hline Male & 128 & 61.5 & 82 & 39.1 & \\
\hline Female & 114 & 54.8 & 96 & 46.4 & \\
\hline Cadre & & & & & $<0.001$ \\
\hline Physician/Clinical Officer & 52 & 41.2 & 73 & 59.2 & \\
\hline Other providers & 183 & 63.0 & 107 & 39.3 & \\
\hline Years delivering TB care & & & & & 0.42 \\
\hline$<5$ years & 132 & 54.2 & 84 & 47.7 & \\
\hline$\geq 5$ years & 111 & 45.7 & 92 & 52.3 & \\
\hline Age in years & 33.8 & $(.9)$ & 33.5 & $(1.4)$ & 0.25 \\
\hline Years of post-basic education & 2.8 & $(.11)$ & 3.2 & $(.12)$ & 0.05 \\
\hline Years providing TB care & 5.8 & $(.63)$ & 7.6 & $(.99)$ & 0.13 \\
\hline
\end{tabular}


Table 6. Multivariate Analysis of Predictors of Performance of D.O.T . (n=286)

\begin{tabular}{|c|c|c|c|}
\hline & Odds Ratio & 95\% C.I. & $p$ Value \\
\hline Cumulative TB Training & 1.2 & $1.0-1.5$ & 0.04 \\
\hline Mean number of sputum tests performed annually & 0.7 & $0.5-1.0$ & $\mathbf{0 . 0 3}$ \\
\hline TB testing offered to STI clients & 2.4 & $0.9-6.2$ & 0.08 \\
\hline Medicine Commodity Score & 1.2 & $1.1-1.4$ & $<0.001$ \\
\hline Supervision Score & 3.2 & $2.0-5.0$ & $<0.001$ \\
\hline
\end{tabular}

Text in bold represent statistically significant findings.

Table 7. Factors Associated with Performance of IPT for HIV+ Clients ( ${ }^{*} n=591$ )

\begin{tabular}{|c|c|c|c|c|c|}
\hline & \multicolumn{5}{|c|}{ Preventive Care for HIV $+n^{*}=591$} \\
\hline & $n^{*}$ & $\%(\mathrm{se})$ & $n^{*}$ & $\%(\mathrm{se})$ & $p$ Value \\
\hline Trained in Prevention of TB (INH) in HIV+ & & & & & $\mathbf{0 . 0 0 7}$ \\
\hline No & 164 & 32.0 & 343 & 68.0 & \\
\hline Yes & 45 & 53.0 & 39 & 47.0 & \\
\hline \multicolumn{6}{|c|}{ Structural Factors } \\
\hline Facility Type & & & & & $<0.001$ \\
\hline Hospital & 140 & 47.4 & 155 & 52.6 & \\
\hline Non-hospital & 69 & 23.3 & 227 & 76.7 & \\
\hline Chest Radiograph performed on-site & & & & & 0.022 \\
\hline No & 70 & 28.5 & 175 & 71.5 & \\
\hline Yes & 123 & 47.7 & 135 & 52.3 & \\
\hline Governmental & 138 & 33.5 & 273 & 66.5 & \\
\hline Non-governmental & 71 & 39.4 & 109 & 60.6 & \\
\hline Facility participates in National DOTS program & & & & & 0.002 \\
\hline No & 47 & 21.2 & 180 & 79.2 & \\
\hline Yes & 162 & 44.5 & 202 & 55.5 & \\
\hline Microscopy provided to STI/HIV clients & & & & & $<0.001$ \\
\hline No & 37 & 17.8 & 170 & 82.3 & \\
\hline Yes & 171 & 44.6 & 212 & 55.3 & \\
\hline Pre-packaged Drugs for Patients & & & & & 0.13 \\
\hline No & 145 & 32.6 & 299 & 67.3 & \\
\hline Yes & 64 & 43.5 & 83 & 56.4 & \\
\hline Supervision score & .84 & $(0.06)$ & .99 & $(0.05)$ & 0.10 \\
\hline Number of clinical duties & 20.8 & $(0.52)$ & 21.2 & $(0.84)$ & 0.12 \\
\hline Physician/Clinical Officer & 52 & 48.0 & 57 & 52.0 & \\
\hline Other providers & 157 & 32.5 & 326 & 67.5 & \\
\hline Years diagnosing OI in HIV+ persons & & & & & 0.01 \\
\hline$<5$ years & 93 & 27.5 & 245 & 72.5 & \\
\hline$\geq 5$ years & 37 & 43.2 & 48 & 56.8 & \\
\hline Years treating $\mathrm{OI}$ in $\mathrm{HIV}+$ persons & & & & & 0.006 \\
\hline$<5$ years & 93 & 25.7 & 269 & 74.2 & \\
\hline$\geq 5$ years & 41 & 42.2 & 55 & 57.8 & \\
\hline Age in years & 35.2 & $(.9)$ & 33.9 & $(1.1)$ & 0.066 \\
\hline Years of post-basic education & 3.2 & $(.09)$ & 2.9 & $(.13)$ & 0.023 \\
\hline Years providing TB care & 5.6 & $(.63)$ & 5.8 & $(1.10)$ & 0.073 \\
\hline Years diagnosing opportunistic infections in HIV+ clients & 4.0 & $(.36)$ & 2.7 & $(.30)$ & $\mathbf{0 . 0 0 1}$ \\
\hline Years managing opportunistic infections in HIV+ clients & 4.1 & $(.36)$ & 2.7 & $(.30)$ & $<\mathbf{0 . 0 0 1}$ \\
\hline
\end{tabular}


Table 8. Multivariate Analysis of Predictors of Provision of IPT to HIV+Clients (n=591)

\begin{tabular}{|l|c|c|c|}
\hline & Odds Ratio & $\mathbf{9 5 \%}$ C.I. & $\boldsymbol{p}$ Value \\
\hline \hline Training in Prevention of OI in HIV+ clients & 0.6 & $0.1-3.6$ & 0.58 \\
\hline Not working in a hospital & 0.8 & $0.4-1.8$ & 0.35 \\
\hline Offering Chest X ray on-site & 0.7 & $0.4-1.4$ & 0.28 \\
\hline Non-participation in the National DOTS Program & 1.8 & $0.6-4.9$ & 0.92 \\
\hline Sputum microscopy performed on site & 1.1 & $0.3-4.5$ & 0.55 \\
\hline TB testing offered to STI clients & 1.3 & $0.6-2.8$ & 0.93 \\
\hline Lab quality score & 1.0 & $0.8-1.2$ & 0.12 \\
\hline Medicine commodity score & 1.1 & $1.0-1.2$ & 0.15 \\
\hline Cadre: mid-level & 0.6 & $0.3-1.2$ & 0.10 \\
\hline Years of post-basic training & 1.34 & $0.9-2.1$ & 0.37 \\
\hline Years providing TB services & 1.2 & $\mathbf{0 . 0 3}$ \\
\hline Age of health worker & $\mathbf{1 . 1}$ & $\mathbf{1 . 0 - 1 . 1}$ & $0.8-2.0$ \\
\hline Supervision score & 0.7 & $0.4-1.1$ & 0.10 \\
\hline Number of clinical duties & 0.9 & & \\
\hline
\end{tabular}

Text in bold represent statistically significant findings.

workers in all sectors. The impact of training in IPT provision for HIV+ clients should be re-assessed now that enabling global policy on IPT for HIV+ patients has been established and training in the 3 Is (Intensified case finding, Infection control, and IPT) is rolling out [60]. Performance of the three key skills, use of smear microscopy, DOT, provision of IPT, are all cost-effective and life saving [61, 62].

Future investments in technical assistance in capacity building should consider the gender equity dimension to assure that trainees reflect the actual TB/HIV workforce. Human resource policies on task-shifting to mid-level providers should be reviewed and updated with the latest evidence [63]. This study reinforces the maxim that highquality $\mathrm{TB}$ diagnosis and treatment require a human resource development strategy, an enabling policy environment, stable access to commodities, laboratory infrastructure, and supportive supervisory structures. The study results reinforce that training and supervision alone are insufficient to dramatically improve staff performance. A strong heath system, including sufficient numbers of staff clear job descriptions, updated guidelines, availability of drugs and appropriate health facility management, is vital to improving health worker capacity and sustaining it over the long term.

$$
\begin{array}{ll}
\text { ABBREVIATIONS } \\
\begin{array}{ll}
\mathrm{AFB} & =\text { Acid-Fast Bacilli } \\
\mathrm{aOR} & =\text { (adjusted) Odds Ratio } \\
\mathrm{CI} & =\text { Confidence Interval } \\
\mathrm{CIDA} & =\text { Canadian International Development Agency } \\
\text { DOT(S) } & =\text { Directly Observed Therapy (, short course) } \\
\mathrm{HIV} & =\text { Human Immunodeficiency Virus } \\
\mathrm{INH} & =\text { isoniazid }
\end{array}
\end{array}
$$

IPT = Isoniazid Preventive Therapy

KSPA $=$ Kenya Service Provisions Assessment

NGO = Non-Governmental Organization

NTP = National Tuberculosis Program

OI $=$ Opportunistic infection

PEPEFAR $=$ Presidents Emergency Plan for AIDS Relief

TB $=$ Tuberculosis

USAID = United States agency for International Development

\section{CONFLICT OF INTEREST}

Funding for data collection was provided by the U.S. Agency for International Development (USAID), the British Department for International Development (DFID), and UNICEF. Both KNCV and PATH are among the NGOs that provided in-service training in TB control at the behest of the Division of Leprosy, Tuberculosis and Lung Disease of the Ministry of Public Health and Sanitation during the 20012004 period. The views expressed in this paper are those of the authors and do not necessarily represent the views of the Government of Kenya, NCAPD, USAID, UNICEF, DFID, the Netherlands Ministry of Foreign Affairs or the organizations with which the authors are affiliated.

\section{ACKNOWLEDGEMENTS}

We thank Bridgette James, Nico Kalisvaart, Kathryn Andersen Clark, and Edine Tiemersma for their technical input on data management and statistical analysis, as well as Verena Mauch for specific insights into the Kenya TB program. The financial support for this analysis from the Netherlands Ministry of Foreign Affairs is gratefully acknowledged. 


\section{REFERENCES}

[1] USAID. The Health Sector Human Resource Crisis in Africa: An Issues Paper. High Level Forum on Health MDGs. Abuja: USAID, Bureau for Africa, Office of Sustainable Development; SARA Project 2004

[2] UNAIDS/WHO. Progress on Global Access to Antiretroviral Therapy: an update on "3 by 5". Geneva.: UNAIDS/WHO. 2005.

[3] Chankova S, Muchiri S, Kombe G. Health workforce attrition in the public sector in Kenya: a look at the reasons. Hum Resour Health 2009; 7: 58.

[4] Dreesch N, Nyoni J, Mokopakgosi O, et al. Public-private options for expanding access to human resources for HIV/AIDS in Botswana. Hum Resour Health 2007; 5: 25.

[5] Dovlo D. Wastage in the health workforce: some perspectives from African countries. Hum Resour Health 2005; 3: 6.

[6] Adano U. The health worker recruitment and deployment process in Kenya: an emergency hiring program. Hum Resour Health 2008; 6: 19.

[7] Wyss K. An approach to classifying human resources constraints to attaining health-related Millennium Development Goals. Hum Resour Health 2004; 2: 11.

[8] McCourt W, Awases M. Addressing the human resources crisis: a case study of the Namibian health service. Hum Resour Health 2007; 5: 1 .

[9] Mathauer I, Imhoff I. Health worker motivation in Africa: the role of non-financial incentives and human resource management tools. Hum Resour Health 2006; 4: 24.

[10] Henderson L, Tulloch J. Incentives for retaining and motivating health workers in Pacific and Asian countries. Hum Resour Health 2008; 6: 18.

[11] Dussault G, Dubois C-A. Human resources for health policies: a critical component in health policies. Hum Resour Health 2003; 1: 1.

[12] Dovlo D. Using mid-level cadres as substitutes for internationally mobile health professionals in Africa. A desk review. Hum Resour Health 2004; $2: 7$.

[13] Vian T, Richards SC, McCoy K, et al. Public-private partnerships to build human capacity in low income countries: findings from the Pfizer program. Hum Resour Health 2007; 5: 8 .

[14] Harries AD, Zachariah R, Bergstrom K, et al. Human resources for control of tuberculosis and HIV-associated tuberculosis. Int J Tuber Lung Dis 2005; 9: 128-37.

[15] Figueroa-Munoz J, Palmer K, Dal Poz M, et al. The health workforce crisis in TB control: a report from high-burden countries. Hum Resour Health 2005; $3: 2$.

[16] Kober K, Van Damme W. Scaling up access to antiretroviral treatment in southern Africa: who will do the job? Lancet 2004; 364: 103-7.

[17] Waako PJ, Odoi-adome R, Obua C, et al. Existing capacity to manage pharmaceuticals and related commodities in East Africa: an assessment with specific reference to antiretroviral therapy. Hum Resour Health 2009; 7: 21.

[18] McCoy D, McPake B, Mwapasa V. The double burden of human resource and HIV crises: a case study of Malawi. Hum Resour Health 2008; 6: 16.

[19] Abuya T, Fegan G, Rowa Y, et al. Impact of ministry of health interventions on private medicine retailer knowledge and practices on anti-malarial treatment in Kenya. Am J Trop Med Hyg 2009; 80: 905-13.

[20] Dräger S, Gedik G, Dal Poz M. Health workforce issues and the Global Fund to fight AIDS, Tuberculosis and Malaria: an analytical review. Hum Resour Health 2006; 4: 23.

[21] Njozing BN, Edin KE, San Sebastian M, et al. Voices from the frontline: counsellors' perspectives on TB/HIV collaborative activities in the Northwest Region, Cameroon. BMC Health Serv Res 2011; 11:328.

[22] Alexander L, Igumbor EU, Sanders D. Building capacity without disrupting health services: public health education for Africa through distance learning. Hum Resour Health 2009; 7: 28.

[23] Uwimana J, Jackson D, Hausler H, et al. Health system barriers to implementation of collaborative TB and HIV activities including prevention of mother to child transmission in South Africa. Trop Med Int Health 2012; 17: 658-65.

[24] Basri C, Bergstrom K, Walton W, et al. Sustainable scaling up of good quality health worker education for tuberculosis control in Indonesia: a case study. Hum Resour Health 2009; 7: 85.
[25] Awofeso N, Schelokova I, Dalhatu A. Training of front-line health workers for tuberculosis control: Lessons from Nigeria and Kyrgyzstan. Hum Resour Health 2008; 6: 20.

[26] Ranson MK, Chopra M, Atkins S, et al. Priorities for research into human resources for health in low- and middle-income countries. Bull World Health Org 2010; 88: 435-43.

[27] Turan JM, Bukusi EA, Cohen CR, et al. Effects of HIV/AIDS on maternity care providers in Kenya. J Obstet Gynecol Neonatal Nurs 2008; 37: 588-95.

[28] Desai M, Rudge JW, Adisasmito W, et al. Critical interactions between Global Fund-supported programmes and health systems: a case study in Indonesia. Health Policy Plan 2010; 25 (Suppl 1): i43-7.

[29] Beaglehole R, Dal Poz M. Public health workforce: challenges and policy issues. Hum Resour Health 2003; $1: 4$.

[30] Kirigia J, Gbary A, Muthuri L, et al. The cost of health professionals' brain drain in Kenya. BMC Health Serv Res 2006; 6: 89.

[31] World Health Organization. A Brief History of Tuberculosis Control in Kenya. Geneva: World Health Organization 2009.

[32] Mansoer J, Scheele S, Floyd K, et al. New methods for estimating the tuberculosis case detection rate in high-HIV prevalence countries: the example of Kenya. Bull World Health Org 2009; 87: 186-92.

[33] Harries AD, Salaniponi FM, Nunn RR, et al. Performance-related allowances within the Malawi National Tuberculosis Control Programme. Int J Tuber Lung Dis. 2005; 9: 138-44.

[34] van't Hoog AH, Onyango J, Agaya J, et al. Evaluation of TB and HIV services prior to introducing TB-HIV activities in two rural districts in western Kenya. Int J Tuber Lung Dis 2008; 12: S32-8.

[35] Nganda B, Wang'ombe J, Floyd K, et al. Cost and costeffectiveness of increased community and primary care facility involvement in tuberculosis care in Machakos District, Kenya. Int J Tuber Lung Dis 2003; 7: S14-20.

[36] Prevention CfDCa. Health worker performance after training in integrated management of childhood illness--Western Province, Kenya, 1996-1997. MMWR Morb Mortal Wkly Rep 1998; 47: 998-1001.

[37] Stanback J, Griffey S, Lynam P, et al. Improving adherence to family planning guidelines in Kenya: an experiment. Int J Qual Health Care 2007; 19: 68-73.

[38] Rowe SY, Kelly JM, Olewe MA, et al. Effect of multiple interventions on community health workers' adherence to clinical guidelines in Siaya district, Kenya. Trans R Soc Trop Med Hyg 2007; 101: 188-202.

[39] Driessche KV, Sabue M, Dufour W, et al. Training health care workers to promote HIV services for patients with tuberculosis in the Democratic Republic of Congo. Hum Resour Health 2009; 7: 23 .

[40] Nsanzumuhire $\mathrm{H}$, Aluoch JA, Karuga WK, et al. A 3rd Study of Case-Finding Methods for Pulmonary Tuberculosis in Kenya, Including the Use of Community Leaders. Tubercle 1981; 62: 7994.

[41] Chakaya JM, Meme H, Kwamanga D, et al. Planning for PPMDOTS implementation in urban slums in Kenya: knowledge, attitude and practices of private health care providers in Kibera slum, Nairobi. Int J Tuber Lung Dis 2005; 9: 403-8.

[42] Hanson C, Kibuga D. Effective tuberculosis control and health sector reforms in Kenya: challenges of an increasing tuberculosis burden and opportunities through reform. Int $\mathrm{J}$ Tuber Lung Dis 2000; 4: 627-32.

[43] Nsanzumuhire H, Lukwago EW, Edwards EA, et al. Study of Use of Community Leaders in Case-Finding for Pulmonary Tuberculosis in Machakos District of Kenya. Tubercle 1977; 58 : 117-28.

[44] Aluoch JA, Karuga WK, Nsanzumuhire H, et al. 2nd Study of Use of Community Leaders in Case-Finding for Pulmonary Tuberculosis in Kenya. Tubercle 1978; 59: 233-43.

[45] Walker DG, Jan S. How do we determine whether community health workers are cost-effective? Some core methodological issues. J Community Health 2005; 30: 221-9.

[46] Chakaya J, Uplekar M, Mansoer J, et al. Public-private mix for control of tuberculosis and TB-HIV in Nairobi, Kenya: outcomes, opportunities and obstacles. Int J Tuber Lung Dis 2008; 12: 1274-8. 
[47] Malotte CK, Morisky DE. Using an unobstrusively monitored comparison study group in a longitudinal design. Health Educ Res 1994; 9: 153-9.

[48] Davis D, O'Brien MA, Freemantle N, et al. Impact of formal continuing medical education: do conferences, workshops, rounds, and other traditional continuing education activities change physician behavior or health care outcomes? JAMA 1999; 282: 867-74.

[49] Thomson O'Brien MA, Freemantle N, Oxman AD, et al. Continuing education meetings and workshops: effects on professional practice and health care outcomes. Cochrane Database Syst Rev 2001; (2): CD003030.

[50] O'Brien MA, Rogers S, Jamtvedt G, et al. Educational outreach visits: effects on professional practice and health care outcomes. Cochrane Database Syst Rev 2007; (4): CD000409.

[51] Lewin S, Dick J, Zwarenstein M, et al. Staff training and ambulatory tuberculosis treatment outcomes: a cluster randomized controlled trial in South Africa. Bull World Health Org 2005; 83: 250-9.

[52] Chakaya JM, Mansoer JR, Scano F, et al. National scale-up of HIV testing and provision of HIV care to tuberculosis patients in Kenya. Int J Tuber Lung Dis 2008; 12: 424-9.

[53] Barr DA. A research protocol to evaluate the effectiveness of public - Private partnerships as a means to improve health and welfare systems worldwide. Am J Public Health 2007; 97: 19-25.

[54] Filerman GL. Closing the management competence gap. Hum Resour Health. 2003; 1: 7.

[55] Dick J, Lewin S, Rose E, et al. Changing professional practice in tuberculosis care: an educational intervention. J Adv Nurs 2004; 48: 434-42.
[56] Jamtvedt G, Young JM, Kristoffersen DT, et al. Does telling people what they have been doing change what they do? A systematic review of the effects of audit and feedback. Qual Saf Health Care 2006; 15: 433-6.

[57] Jamtvedt G, Young JM, Kristoffersen DT, et al. Audit and feedback: effects on professional practice and health care outcomes. Cochrane Database Syst Rev 2006; (2): CD000259.

[58] National Coordinating Agency for Population and Development [Kenya], Ministry of Health CBoS, Macro International, National, Coordinating Agency for Population and Development [Kenya] Mo, et al. Kenya Service Provision Assessment Survey 2004 Nairobi, Kenya: Nairobi, Kenya 2005.

[59] Ferrinho P, Van Lerberghe W, Fronteira I, et al. Dual practice in the health sector: review of the evidence. Hum Resour Health 2004; $2: 14$.

[60] WHO DoHASTD. Guidelines for intensified tuberculosis casefinding and isoniazid preventive therapy for people living with HIV in resource-constrained settings. Report No.: ISBN 978924 150070 8. Geneva: WHO 2010, December 2010.

[61] Wilkinson D, Squire SB, Garner P. Effect of preventive treatment for tuberculosis in adults infected with HIV: systematic review of randomised placebo controlled trials. Br Med J 1998; 317: 625-9.

[62] Currie CSM, Floyd K, Williams BG, et al. Cost, affordability and cost-effectiveness of strategies to control tuberculosis in countries with high HIV prevalence. BMC Public Health 2005; 5: 130.

[63] Gericke CA, Kurowski C, Ranson $\mathrm{MK}$, et al. Intervention complexity - a conceptual framework to inform priority-setting in health. Bull World Health Org 2005; 83: 285-93.

(C) Mitchell et al.; Licensee Bentham Open.

This is an open access article licensed under the terms of the Creative Commons Attribution Non-Commercial License (http: //creativecommons.org/licenses/by$\mathrm{nc} / 3.0 /$ ) which permits unrestricted, non-commercial use, distribution and reproduction in any medium, provided the work is properly cited. 